noninvasive means of predicting response to chemotherapy in patients with advanced bladder cancer, allowing nonresponders to be identified early on, and spared ineffective treatment and associated morbidity.

Tamsin Osborne

Original article Schrier BP et al. (2006) Evaluation of chemotherapy with magnetic resonance imaging in patients with regionally metastatic or unresectable bladder cancer. Eur Urol 49: 698-703

\section{Intermittent androgen ablation is an effective secondary therapy for prostate cancer}

Continuous androgen suppression is the most common secondary therapy for biochemical failure in patients who have undergone radiotherapy for localized prostate cancer, but can result in the recurrence of the tumor in an androgen-independent form. It has been suggested that intermittent androgen ablation (IAA) could delay the development of hormone resistance in prostate tumors, as well as having less-severe side effects than continuous hormone deprivation.

In a phase II study, researchers in Canada assessed the effects of IAA in 39 patients with biochemical failure after treatment with radiotherapy for prostate cancer. Patients were given the luteinizing-hormone-releasing hormone analog buserelin at 2-monthly intervals for 8 months, after which serum prostate-specific antigen and testosterone levels were assessed every 4-6 months. Another cycle of treatment was given if the patient's prostate-specific antigen level rose above $10 \mathrm{ng} / \mathrm{ml}$. The median time between the first and second cycle was 20.1 months, dropping to 15.5 months between the third and fourth cycles. Four patients developed androgen resistance. The 5-year survival rate was $92.3 \%$. Hot flashes were a common side effect. Although generally well tolerated, treatment did have an impact on patient's quality of life; however, with testosterone recovery between cycles, symptoms were alleviated.

The authors conclude that IAA is a safe and effective treatment, with a similar effect on prostate-specific antigen levels to continuous androgen ablation, but producing less-severe side effects. They also note the $66 \%$ saving in cost of treatment compared with continuous therapy.

Jim Casey
Original article Cury FLB et al. (2006) Intermittent androgen ablation in patients with biochemical failure after pelvic radiotherapy for localized prostate cancer. Int J Radiat Oncol Biol Phys 64: 842-848

\section{A noninvasive proteomic method for early detection of renal allograft rejection}

Researchers in Germany have found that analysis of urine proteins using SURFACE-ENHANCED LASER DESORPTION/IONIZATION TIME-OF-FLIGHT MASS SPECTROMETRY (SELDI-TOF-MS) can be used to reliably diagnose acute allograft rejection in kidney transplant recipients. This noninvasive tool might be preferable to renal biopsy, the current gold-standard diagnostic method.

The retrospective analysis included 23 renal transplant recipients with clinical symptoms of rejection, including increased graft volume, proteinuria and decreased diuresis. Of these patients, renal biopsy revealed that only 13 actually had acute rejection. Midstream urine samples from all subjects were analyzed using two ProteinChip ${ }^{\circledR}$ (Ciphergen Biosystems Inc., Fremont, CA) arrays with different chromatographic surfaces, followed by SELDI-TOF-MS. Cluster-and-rule-based data mining identified two biomarkers that had high diagnostic sensitivity and specificity for biopsy-proven acute rejection. The researchers proposed that these two biomarker proteins (molecular weights $25,708 \mathrm{kDa}$ and $28,133 \mathrm{kDa}$ ) might be GLUTATHIONE-S-TRANSFERASE and TISSUE PLASMINOGEN ACTIVATOR. Further investigation is required to validate these findings, identify other potential biomarkers and improve our understanding of the pathology of acute allograft rejection.

SELDI-TOF-MS-based ProteinChip ${ }^{\circledR}$ analysis is less expensive and more convenient than renal biopsy; it is also quicker and requires smaller sample volumes than other mass spectrometry techniques. The SELDI-TOF-MSbased ProteinChip ${ }^{\circledR}$ technology might be used in future to monitor the immunological reaction between recipient and graft, before the graft becomes damaged. This new technique would aid early treatment with corticosteroids and improve prognosis.

\section{Rachael Williams}

Original article Reichelt O et al. (2006) Prediction of renal allograft rejection by urinary protein analysis using ProteinChip arrays (surface-enhanced laser desorption/ionization time-offlight mass spectrometry). Urology 67: 472-475

\section{GLOSSARY}

SURFACE-ENHANCED LASER DESORPTION/ IONIZATION TIMEOF-FLIGHT MASS SPECTROMETRY (SELDITOF-MS)

Technology that combines mass spectrometry with varying affinitychromatographic chips

GLUTATHIONE-STRANSFERASE An enzyme that protects cells against the potential toxicity of a number of compounds

TISSUE PLASMINOGEN ACTIVATOR

An enzyme that generates plasmin from plasminogen; produced by both normal and invasive cells 